\title{
La brecha de las noticias en Argentina: factores contextuales y preferencias de periodistas y público
}

\section{Eugenia Mitchelstein ${ }^{1}$ \\ Pablo J. Boczkowski² \\ Celeste Wagner ${ }^{3}$ \\ Silvana Leiva ${ }^{4}$}

Recibido: 2016-08-25

Enviado a pares: 2016-09-01
Aprobado por pares: 2016-09-21

Aceptado: 2016-09-23

\section{DOI: 10.5294/pacla.2016.19.4.4}

Para citar este artículo / to reference this article / para citar este artigo

Mitchelstein, E., Boczkowski, P.J., Wagner, C. y Leiva, S. (2016). La brecha de las noticias en Argentina: factores contextuales y preferencias de periodistas y público. Palabra Clave, 19(4), 1027-1047. DOI: 10.5294/pacla.2016.19.4.4

\section{Resumen}

Esta investigación examina la oferta y la demanda de noticias en siete sitios de la Argentina a través de un análisis de contenido cuantitativo de las noticias publicadas de manera más prominente en las homepages o páginas de inicio y las listas de las "más leídas" durante un periodo de siete meses, que incluye la campaña presidencial y la asunción de un nuevo presidente en 2015. El análisis cuantitativo de más de 5500 noticias muestra que existe una brecha significativa y consistente entre las preferencias de los periodistas y los consumidores y una mayor variabilidad en las preferencias de los integrantes del público que en las elecciones de los productores profesionales de noticias. Este trabajo utiliza tales hallazgos para reflexionar sobre los conceptos de fijación de agenda y ciudadanía monitorial.

\footnotetext{
Universidad de San Andrés. Argentina. emitchelstein@udesa.edu.ar

2 Northwestern University. Estados Unidos.pjb9@northwestern.edu

3 University of Pennsylvania. Estados Unidos. celeste.wagner@asc.upenn.edu

$4 \quad$ Universidad de San Andrés. Argentina. sleiva@udesa.edu.ar
} 


\section{Palabras clave}

Periodismo en línea; análisis del contenido; Consumo de noticias; Argentina; Comunicación política; Audiencias, Establecer la agenda (Fuente: Tesauro de la Unesco).

\section{The News Gap in Argentina: Contextual Factors and Preferences of Journalists and the Audience}

\section{Abstract}

This investigation examines the supply and demand of news in seven places in Argentina through a quantitative content analysis of the news more prominently published on homepages and "most read" lists over a period of seven months, including the presidential campaign and the 2015 presidential inauguration. The quantitative analysis of over 5500 news shows that there is a significant and consistent gap between the preferences of journalists and consumers and a greater variability in the preferences of members of the public than in the choice of professional news producers. This paper uses these findings to reflect on the concepts of agenda-setting and monitorial citizenship.

\section{Keywords}

Online journalism; content analysis; news consumption; Argentina; political communication; audiences; agenda-setting (Source: Unesco Thesaurus). 


\section{A brecha das notícias na Argentina: fatores contextuais e preferências de jornalistas e público}

\section{Resumo}

Esta pesquisa examina a oferta e a demanda de notícias em sete lugares da Argentina através de uma análise de conteúdo quantitativo das notícias publicadas de maneira mais proeminente nas homepages ou páginas de início e as listas das "mais lidas" durante um período de sete meses, que inclui a campanha presidencial e a assunção de um novo presidente em 2015. A análise quantitativa de mais de 5500 notícias mostra que existe una brecha significativa e consistente entre as preferências dos jornalistas e dos consumidores e uma maior variabilidade nas preferências dos integrantes do público do que nas eleições dos produtores profissionais de notícias. Este trabalho utiliza estas descobertas para refletir sobre os conceitos de agendamento e cidadania monitorial.

\section{Palavras-chave}

Jornalismo digital; análise de conteúdo; consumo de notícias; Argentina; comunicação política; audiências; agenda setting (Fonte: Tesauro da Unesco). 


\section{Introducción}

Los medios cumplen al menos dos funciones fundamentales en regímenes democráticos: proveen de información a los ciudadanos para la toma de decisiones (Bennett y Entman, 2001; Downs, 1957) y ofrecen una agenda de temas a los que gobernados y gobernantes deberían prestar atención (McCombs y Shaw, 1972; Scheufele y Tewksbury, 2007). Sin embargo, la literatura sugiere que, mientras editores y periodistas tienden a dar prioridad a las noticias sobre asuntos públicos, como política nacional e internacional y economía y negocios, los consumidores, en cambio, prefieren noticias sobre asuntos no públicos, como deportes, espectáculos, policiales y el clima. Esta divergencia temática resulta en una brecha entre la oferta y la demanda de información de actualidad (Boczkowski y Mitchelstein, 2013).

Esta investigación examina la oferta y la demanda de noticias en siete sitios de la Argentina a través de un análisis de contenido cuantitativo de las noticias publicadas de manera más prominente en las homepages o páginas de inicio y las listas de las "más leídas" durante un periodo de siete meses (entre septiembre de 2015 y marzo de 2016). Este lapso incluye los meses finales de la campaña presidencial (los comicios por la primera vuelta fueron el 25 de octubre, y por el balotaje, o segunda vuelta, el 22 de noviembre) y la asunción del nuevo presidente, Mauricio Macri, el 10 de diciembre. Además, durante el periodo de recolección de datos, se fugaron de una cárcel de máxima seguridad tres condenados por el homicidio de tres empresarios vinculados al narcotráfico, acusados de haber donado dinero a la campaña presidencial de la expresidenta Cristina Fernández de Kirchner. Estuvieron prófugos 17 días, entre el 27 de diciembre de 2015 y el 11 de enero de 2016. Estos tres eventos —elección presidencial, asunción del nuevo primer mandatario y triple fuga - permiten a esta investigación examinar la posible variación en los niveles de noticias sobre asuntos públicos tanto entre la selección de los periodistas (representada por las noticias más visibles en las páginas de inicio de los siete sitios) como en las preferencias de los consumidores (visibles en las listas de "más leídas” o "más cliqueadas"). 
El análisis muestra que existe una brecha significativa y consistente entre las preferencias de los periodistas y los consumidores durante un periodo que incluye una campaña presidencial y la fuga de la cárcel de tres condenados por homicidio con conexiones con el poder político. Además, indica que hay una mayor variabilidad en las preferencias de los integrantes del público que en las elecciones de los productores profesionales de noticias. Finalmente, los datos incluyen una notable excepción a la tendencia de la brecha: el mes de diciembre de 2015, en el que la brecha entre periodistas y consumidores desaparece.

Este trabajo se apoya en tales hallazgos para reflexionar sobre los conceptos de fijación de agenda y ciudadanía monitorial en la nueva matriz de comunicación digital, en la que los consumidores incurren en menores costos en la selección tanto de las fuentes de información como (dentro de esos medios) de las noticias que les interesan.

\section{Consideraciones teóricas}

Una de las principales funciones de los medios de comunicación es proveer a los lectores de información esencial para el funcionamiento del cuerpo político (Druckman, 2005; Graber, 2006; Iyengary Kinder, 1987). Sin embargo, la literatura sugiere que existe una brecha entre las noticias que editores y periodistas prefieren publicar y las que las audiencias eligen leer. Los periodistas, tanto por formación profesional (Cook, 1998; Davis, 1995; Williams y Delli Carpini, 2012) como por requisitos organizacionales (Bennett, Lawrence y Livingston, 2008; Gitlin, 1980), tienden a privilegiar noticias sobre política, economía y asuntos internacionales ("asuntos públicos"), por sobre los artículos acerca de temas policiales, entretenimiento o deportes ("asuntos no públicos”) (Gans, 2004; Jones, 2009; Tuchman, 1978).

Una corriente de investigación sugiere que, en cambio, las audiencias no suelen estar tan interesadas por los asuntos públicos como los periodistas (Bird, 2003; Hamilton, 2004; Prior, 2007; Tewksbury, 2003). Estos hallazgos son congruentes con el concepto de ignorancia racional de Downs (1957), que explica que los ciudadanos no invierten recursos en informarse sobre un tema cuando consideran que el costo de hacerlo es más alto que 
los beneficios percibidos de esa actividad. Otra corriente, por el contrario, ha sostenido que los consumidores de noticias están principalmente interesados por asuntos de opinión pública (Dean, Pertilla y Bert, 2007; Patterson, 1994; Shoemaker y Cohen, 2006) y que incluso a veces sienten que es un deber como ciudadanos el estar informados en estos temas (Huang, 2009). La literatura sobre el comportamiento de las audiencias frente a la oferta del periodismo es, entonces, profusa aunque divergente.

Como señalan Boczkowski y Mitchelstein (2013, p. 9), tradicionalmente la literatura ha estudiado las preferencias de los periodistas y la de los ciudadanos por separado, producto de la "división del trabajo" entre los académicos. Los estudios sobre periodismo han dado cuenta de la oferta noticiosa de los medios, mientras que la literatura sobre audiencias ha investigado el comportamiento de los consumidores de noticias. Por otra parte, mientras que hasta hace pocos años las preferencias de las audiencias solo se podían estudiar de manera agregada e indirecta (a través de información sobre circulación y ratings, o encuestas y focus groups), los medios digitales permiten conocer de manera más precisa el comportamiento de las audiencias.

La idea de una "brecha noticiosa" entre periodistas y audiencias no es nueva: puede rastrearse, al menos, setenta y cinco años atrás, cuando Robert Park, uno de los principales exponentes de la Escuela de Sociología de Chicago, que además había trabajado como periodista, escribió: "Las cosas que más nos gustarían publicar no son aquellas cosas que queremos leer. Lo que queremos obtener en los medios es información 'edificante', pero lo que queremos leer es algo 'interesante'” (Park, 1981). El significado de esta brecha cambia según el contexto. La lógica del mercado de los grandes medios tradicionales, durante casi todo el siglo XX, le permitía a estos decirle al público qué información debían tener, de manera relativamente independiente de sus preferencias (Schudson, 1978; Zelizer, 2004). Restricciones estructurales como las frecuencias disponibles en el espectro radioeléctrico y los altos costos de ingreso en el negocio limitaban el número de competidores por la atención del público. A su vez, la lógica del formato de estos medios hacía que la capacidad de discriminación de noticias por parte de 
las audiencias estuviese muy restringida: para enterarse de las novedades deportivas había que escuchar el noticiero entero o pasar las páginas del diario hasta la sección de deportes. Si no voluntario, por lo menos el consumo de ciertas noticias de asuntos públicos resultaba siendo "accidental". En un contexto de escasa competencia, esta condición permitió a las organizaciones de medios conservar una misión de servicio público que gozaba a la vez de altos márgenes de rentabilidad económica.

Actualmente, en un contexto donde los medios digitales son cada vez más dominantes, tanto periodistas como analistas del fenómeno son cada vez más conscientes de que los consumidores no fijan su atención en aquellos temas que la literatura ha considerado tradicionalmente "esenciales" para el desarrollo de una democracia liberal. Tanto cuestiones de competencia como de formato de los medios digitales habilitan a los públicos a ignorar lo que no les interesa de manera muy sencilla y a consumir lo que sí les interesa del mismo modo. A esta diferencia entre lo que los medios y periodistas ofrecen - lo que consideran "más importante" de acuerdo con su ética profesional moderna-y lo que los lectores o consumidores finalmente prefieren se ha llamado la "brecha de las noticias" (Boczkowski y Mitchelstein, 2013).

Estudiar la brecha entre la oferta y la demanda de noticias permite echar luz sobre dos herramientas conceptuales muy usadas en el campo de los estudios de periodismo y opinión pública: la fijación de agenda (McCombs y Shaw, 1972) y la noción de ciudadanía monitorial (Schudson, 1998). De acuerdo con la teoría de la fijación de agenda, los medios le sugieren a las audiencias cuáles son los temas, las personas y los eventos a los que vale la pena prestarles más atención. Como consecuencia de este proceso, existiría una correspondencia entre el énfasis que los medios le dan a ciertos temas y la importancia que les otorga la ciudadanía (McCombs y Stroud, 2014). Sin embargo, las investigaciones previas sugieren que la capacidad de fijar agenda depende del contexto, que podría moderar el poder que los medios líderes tienen para configurar la agenda (Boczkowski y Mitchelstein, 2013; Boczkowski, Mitchelstein y Walter, 2012). Esto en gran medida se puede explicar a partir de la noción de ciudadanía monitorial (Schudson, 
1998), que indica que el público no suele seguir cuidadosamente los temas de asuntos públicos en momentos de rutina, sino que habitualmente tiene una mirada superficial sobre las noticias, y solo fija su atención en estos temas cuando cuestiones más extraordinarias alteran la dinámica de la política o la sociedad (como los casos de las campañas electorales o crisis políticas de envergadura) (Cho, 2008; Tewskbury, 2006).

La existencia de una divergencia entre lo que los periodistas priorizan y las audiencias eligen pone en cuestión dos roles tradicionales del periodismo: ser proveedores de información y generar espacios de deliberación pública. En la medida en que las audiencias pierden interés en los asuntos públicos, existe una ciudadanía que, bien no está preparada, bien no está interesada en hablar de temas que son esenciales para la vida democrática. Este escenario de fragmentación es también para los medios un desincentivo para ejercer sus funciones tradicionales de control sobre la política y de exigencia de rendición de cuentas. En este sentido, estudiar la divergencia entre la oferta y la demanda de noticias no es relevante únicamente por el problema que supone para la supervivencia de los medios en su formato más tradicional, sino que también pone en cuestión la matriz de comunicación pública y actividad política que se sostuvo durante casi todo el siglo XX y que animó ciertas visiones de las sociedades democráticas y liberales.

\section{Método}

Para estudiar la brecha noticiosa, hemos realizado análisis de contenido en los siguientes siete sitios en línea de noticias argentinos: Clarín.com (diario Clarín ${ }^{5}$ ), Lanacion.com.ar (diario La Nación ${ }^{6}$ ), Perfil.com (diario Perfil $\left.{ }^{7}\right)$, Infobae.com $\left(\right.$ Infobae $\left.{ }^{8}\right)$, TN.com.ar (canal de noticias $\mathrm{TN}^{9}$ ),

5 Clarín se publica en Buenos Aires y tiene una circulación diaria promedio de 233000 ejemplares (IVC, 2016).

6 La circulación diaria promedio de La Nación, también publicado en Buenos Aires, es de 139000 (IVC, 2016).

7 Diario Perfil se publica sábados y domingos en Buenos Aires, con una circulación aproximada de 27000 ejemplares (IVC, 2016).

8 Infobae es el único sitio de la muestra que solo se publica en internet, y es uno de los sitios de noticias más visitados, junto con Clarín y La Nación.

9 Todo Noticias (TN) es la versión en línea del sitio de noticias por cable con el rating más alto de la Argentina (Ibope, 2016). 
Lavoz.com.ar (diario $L a V o z^{10}$ ) y Diariouno.com.ar (diario Uno ${ }^{11}$ ). Los datos se recolectaron en 47 días desde septiembre 2015 hasta marzo $2016 .{ }^{12}$ La selección de estos sitios en línea responde a varias razones. En primer lugar, una buena parte de estos sitios se encuentra entre los sitios más leídos de la Argentina, poseen un alcance nacional en sus publicaciones y un lugar prominente en la fijación de agenda. En segundo lugar, dos de los sitios corresponden a diarios de referencia en provincias o regiones argentinas. En tercer lugar, todos estos sitios reportan públicamente información sobre el consumo de sus lectores, es decir, listas de las notas más leídas y, en algunos casos, las más comentadas y más compartidas. En todos los casos, se trata de diarios denominados "generalistas", en la medida en que combinan, en su oferta, noticias sobre asuntos públicos con noticias sobre esparcimiento, cultura, educación, deportes, etc. Por esta razón, estos sitios concentran una audiencia heterogénea y masiva. Esta heterogeneidad es útil en esta investigación en la medida en que nos permite evaluar las diferencias y similitudes entre la elección de los periodistas y la de los consumidores a la hora de elegir noticias.

La información que hemos reunido responde a las dos partes de la "brecha" que nos interesa analizar: la elección de los periodistas y la de sus audiencias. Para ello, seleccionamos, en cada día de recolección de datos, las diez primeras noticias que aparecieron en la homepage de cada uno de los sitios, siguiendo el orden de izquierda a derecha y de arriba abajo (de aquí en adelante, la "lista de los periodistas"). Seleccionamos las primeras diez noticias presentes en la homepage de cada diario, porque entendemos que aquellas que aparecen en la parte superior del sitio son las consideradas más "importantes" por los periodistas y por ende son un indicador de lo más noticioso para el periodismo. Complementario a esto, recolectamos para cada día de recolección la lista de noticias más leídas, según la información reportada por cada sitio (de aquí en adelante "las más leídas").

10 La Voz del Interior se publica en Córdoba y tiene una circulación diaria aproximada de 30000 ejemplares (IVC, 2016).

11 Diario Uno se publica en Mendoza y tiene una circulación diaria aproximada de 11000 ejemplares (IVC, 2016).

12 La muestra incluye seis días de septiembre, ocho días de octubre, cinco de noviembre, siete de diciembre, siete de enero, siete de febrero y siete de marzo. 
La unidad de análisis de este estudio es la noticia. Las variables que codificamos fueron las siguientes:

- Lista:

- De periodistas.

- De consumidores.

- Contenido:

- Asuntos públicos: una noticia fue categorizada como de asuntos públicos si involucraba temas de economía, de gobierno, de política, de negocios, relaciones internacionales, o si involucraba a figuras públicas o tenía una implicancia política explícita.

- Asuntos no públicos: noticias sobre deportes, entretenimiento, crimen, tecnología, cultura, educación, el clima o toda noticia que no tuviera ningún tipo de vinculación con los asuntos públicos.

Esta segunda variable busca dilucidar la existencia y naturaleza de una brecha en las preferencias temáticas de periodistas y consumidores, y la categorización está basada en el libro de Boczkowski y Mitchelstein (2013).

Como la brecha de las noticias es sensible al paso del tiempo y presenta cambios en función del nivel de actividad política que se presente, hemos recolectado información tanto en periodos de actividad política normal como periodos de alto nivel de actividad política. como la campaña presidencial argentina en 2015 y la posterior asunción del nuevo presidente, Mauricio Macri.

En total, se han analizado 5598 noticias, de las cuales $3251^{13}$ pertenecen a la lista de las noticas privilegiadas por los periodistas, mientras que las 2347 restantes pertenecen a la lista de las preferencias de los consumidores. ${ }^{14}$ Siete asistentes de investigación codificaron las noticias durante los

13 La diferencia con el número esperado de noticias de siete sitios en 47 días (3290) es de 1\% y se debe a errores de los asistentes de investigación en la recolección de datos.

14 La diferencia entre la cantidad de noticias examinadas en la lista de "periodistas" y la de "consumidores" se debe a que no todos los sitios publicaban diez noticias en la lista de las más leídas.

1036 La brecha de las noticias en Argentina: factores contextuales y preferencias... - Eugenia Mitchelstein y otros 
siete meses que abarca el estudio. La fiabilidad intercodificadores o índice kappa de Cohen fue calculado sobre $19 \%$ de la muestra (9 días) y el promedio de todas las variables fue 0.80 .

\section{Hallazgos}

El análisis de la cobertura noticiosa de los siete medios analizados durante el periodo septiembre de 2015 a marzo de 2016 indica que, si bien la brecha respecto de los temas de asuntos públicos es constante, disminuye en periodos de alta actividad política, es decir, depende de la coyuntura. En los meses de fuerte actividad política, como los meses finales de la campaña presidencial (septiembre-noviembre de 2015), la brecha noticiosa entre noticias de asuntos públicos y noticias de asuntos no públicos muestra una disminución notable (figura 1). En octubre, mes de la primera vuelta de la elección presidencial, el interés de las audiencias por los temas de los asuntos públicos aumentó, pero nunca el consumo de temas de política, economía y relaciones internacionales llegó a convertirse en la mayoría de las noticias más leídas, excepto en dos sitios ( La Voz y Perfil) (cuadro 1). En casi todos los sitios, las audiencias prefirieron consumir noticias sobre información general, deportes, espectáculos, entretenimiento, policiales y ciencia y tecnología. Aproximadamente, la brecha entre los artículos publicados por los periodistas de manera más prominente y las noticias que aparecen en la lista de las más leídas fue de 14 puntos porcentuales durante el periodo analizado.

En los meses finales de la campaña, se puede observar un aumento tanto en la oferta como en la demanda de noticias sobre la campaña electoral, aunque el interés del público fue siempre menor que el de los periodistas. El tipo de campaña política, en la que dos candidatos competían con posibilidades de ser electos presidente, $y$ la existencia de una segunda vuelta o balotaje fueron elementos todos que mantuvieron tanto a periodistas como a lectores muy cercanos a la actualidad política. Frente a la campaña, los ciudadanos monitoriales, alertados por los medios de que la elección requería su atención, aumentaron su consumo de noticias sobre asuntos públicos. 


\section{Figura 1. Porcentaje de noticias de asuntos públicos en los siete sitios examinados, de septiembre a marzo de 2016}

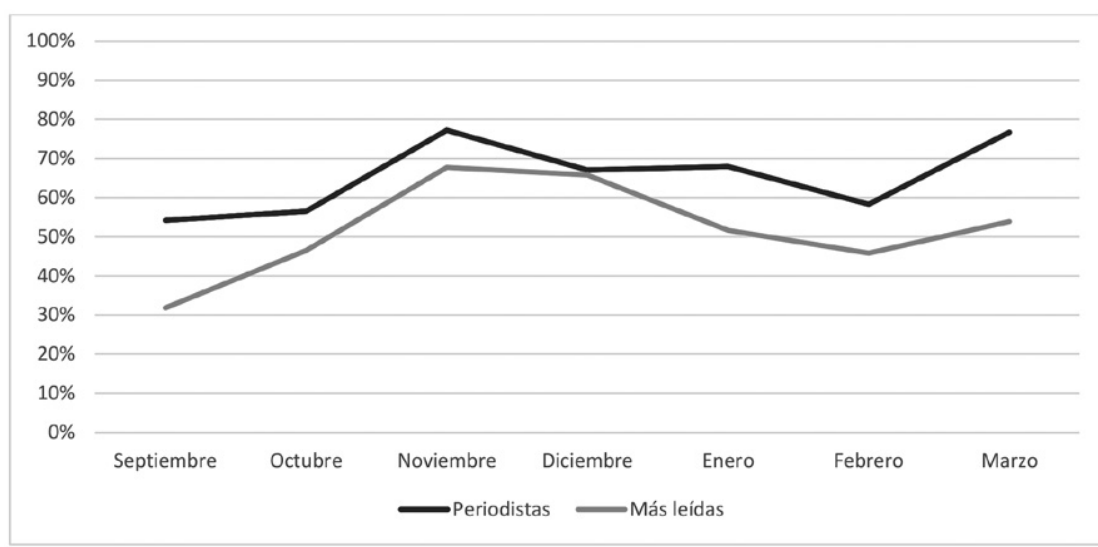

\section{Cuadro 1. Porcentaje de noticias de asuntos públicos en las listas de periodistas y consumidores entre septiembre y diciembre de 2015}

\begin{tabular}{|c|c|c|c|c|c|c|c|c|}
\hline & \multicolumn{2}{|c|}{ Septiembre } & \multicolumn{2}{|c|}{ Octubre } & \multicolumn{2}{|c|}{ Noviembre } & \multicolumn{2}{|c|}{ Diciembre } \\
\hline & Periodistas & Consumidores & Periodistas & Consumidores & Periodistas & Consumidores & Periodistas & Consumidores \\
\hline \multirow[t]{2}{*}{ Clarín } & $65 \%$ & $23.73 \%$ & $54 \%$ & $35 \%$ & $82.00 \%$ & $70.00 \%$ & $63.24 \%$ & $62.69 \%$ \\
\hline & \multicolumn{2}{|c|}{$\begin{array}{c}\operatorname{chi} 2(1)=20.5128 \\
\operatorname{Pr}=0.000\end{array}$} & \multicolumn{2}{|c|}{$\begin{array}{c}\operatorname{chi} 2(1)=5.6971 \\
\operatorname{Pr}=0.017\end{array}$} & \multicolumn{2}{|c|}{$\begin{array}{c}\operatorname{chi} 2(1)=1.9737 \\
\operatorname{Pr}=0.160\end{array}$} & \multicolumn{2}{|c|}{$\begin{array}{c}\operatorname{chi} 2(1)=0.0044 \\
\operatorname{Pr}=0.947\end{array}$} \\
\hline \multirow[t]{2}{*}{$\begin{array}{c}\text { La } \\
\text { Nación }\end{array}$} & $59.32 \%$ & $34.29 \%$ & $73.75 \%$ & $45.00 \%$ & $94.00 \%$ & $84.00 \%$ & $86.96 \%$ & $88.89 \%$ \\
\hline & \multicolumn{2}{|c|}{$\begin{array}{c}\operatorname{chi} 2(1)=5.5080 \\
\operatorname{Pr}=0.019\end{array}$} & \multicolumn{2}{|c|}{$\begin{array}{c}\operatorname{chi} 2(1)=9.5862 \\
\operatorname{Pr}=0.002\end{array}$} & \multicolumn{2}{|c|}{$\begin{array}{c}\operatorname{chi} 2(1)=1.9695 \\
\operatorname{Pr}=0.160\end{array}$} & \multicolumn{2}{|c|}{$\begin{array}{c}\operatorname{chi} 2(1)=1.9695 \\
\operatorname{Pr}=0.160\end{array}$} \\
\hline \multirow[t]{2}{*}{ Perfil } & $68.33 \%$ & $72.73 \%$ & $68 \%$ & $91 \%$ & $88 \%$ & $86 \%$ & $86 \%$ & $90 \%$ \\
\hline & \multicolumn{2}{|c|}{$\begin{array}{c}\operatorname{chi} 2(1)=0.2660 \\
\operatorname{Pr}=0.606\end{array}$} & \multicolumn{2}{|c|}{$\begin{array}{c}\operatorname{chi} 2(1)=1.9695 \\
\operatorname{Pr}=0.160\end{array}$} & \multicolumn{2}{|c|}{$\begin{array}{c}\operatorname{chi} 2(1)=0.0884 \\
\operatorname{Pr}=0.766\end{array}$} & \multicolumn{2}{|c|}{$\begin{array}{c}\operatorname{chi} 2(1)=0.6026 \\
\operatorname{Pr}=0.438\end{array}$} \\
\hline \multirow[t]{2}{*}{ Infobae } & $66.10 \%$ & $38.46 \%$ & $60 \%$ & $33 \%$ & $79 \%$ & $72 \%$ & $61 \%$ & $74 \%$ \\
\hline & \multicolumn{2}{|c|}{$\begin{array}{c}\operatorname{chi} 2(1)=5.6471 \\
\operatorname{Pr}=0.017\end{array}$} & \multicolumn{2}{|c|}{$\begin{array}{c}\operatorname{chi} 2(1)=8.0689 \\
\operatorname{Pr}=0.005\end{array}$} & \multicolumn{2}{|c|}{$\begin{array}{c}\operatorname{chi} 2(1)=0.4726 \\
\operatorname{Pr}=0.492\end{array}$} & \multicolumn{2}{|c|}{$\begin{array}{c}\operatorname{chi} 2(1)=1.7120 \\
\operatorname{Pr}=0.191\end{array}$} \\
\hline \multirow[t]{2}{*}{$\mathrm{TN}$} & $46.67 \%$ & $10.00 \%$ & $57.50 \%$ & $37.88 \%$ & $62.00 \%$ & $71.11 \%$ & $58.57 \%$ & $67.19 \%$ \\
\hline & \multicolumn{2}{|c|}{$\begin{array}{c}\operatorname{chi} 2(1)=19.8632 \\
\operatorname{Pr}=0.000\end{array}$} & \multicolumn{2}{|c|}{$\begin{array}{c}\operatorname{chi} 2(1)=5.5734 \\
\operatorname{Pr}=0.018\end{array}$} & \multicolumn{2}{|c|}{$\begin{array}{c}\operatorname{chi} 2(1)=0.8802 \\
\operatorname{Pr}=0.348\end{array}$} & \multicolumn{2}{|c|}{$\begin{array}{c}\operatorname{chi} 2(1)=1.0611 \\
\operatorname{Pr}=0.303\end{array}$} \\
\hline \multirow[t]{2}{*}{$\mathrm{La} \mathrm{Voz}$} & $48.33 \%$ & $30.56 \%$ & $41 \%$ & $39 \%$ & $78.00 \%$ & $56.00 \%$ & $62.86 \%$ & $47.63 \%$ \\
\hline & \multicolumn{2}{|c|}{$\begin{array}{c}\operatorname{chi} 2(1)=2.9257 \\
\operatorname{Pr}=0.087\end{array}$} & \multicolumn{2}{|c|}{$\begin{array}{c}\operatorname{chi} 2(1)=0.0557 \\
\operatorname{Pr}=0.813\end{array}$} & \multicolumn{2}{|c|}{$\begin{array}{c}\operatorname{chi} 2(1)=3.8915 \\
\operatorname{Pr}=0.049\end{array}$} & \multicolumn{2}{|c|}{$\begin{array}{c}\operatorname{chi} 2(1)=2.5236 \\
\operatorname{Pr}=0.112\end{array}$} \\
\hline \multirow[t]{2}{*}{ Uno } & $25 \%$ & $15 \%$ & $43 \%$ & $10 \%$ & $56 \%$ & $12 \%$ & $50.72 \%$ & $11.11 \%$ \\
\hline & \multicolumn{2}{|c|}{$\begin{array}{c}\operatorname{chi} 2(1)=1.4467 \\
\operatorname{Pr}=0.229\end{array}$} & \multicolumn{2}{|c|}{$\begin{array}{c}\operatorname{chi} 2(1)=13.2909 \\
\operatorname{Pr}=0.000\end{array}$} & $\begin{array}{r}\text { chi2 }(1 \\
\operatorname{Pr}\end{array}$ & $\begin{array}{l}=13.3065 \\
=0.000\end{array}$ & & $\begin{array}{l}9008 \\
0.000\end{array}$ \\
\hline
\end{tabular}


Aunque en diciembre de 2015 la campaña había llegado a su fin, la atención hacia los asuntos públicos por parte de la audiencia no decayó y la brecha casi desapareció; la diferencia entre el porcentaje de noticias de asuntos públicos en la lista de los periodistas y en la lista de las más leídas no es estadísticamente significativa si consideramos todos los sitios en agregado. Este hallazgo tiene que ver, entre otras cosas, con el hecho de que la resolución de la campaña política dejó como ganador al partido opositor, con lo que se generó una situación de alternancia en dos niveles importantes: nacional, luego de catorce años, y en la provincia de Buenos Aires, luego de veintiocho años. Estos cambios generaron nuevas expectativas, alimentadas en parte por la agenda de cambio promocionada por el partido ganador. En síntesis, la llegada de un nuevo Gobierno y el anuncio de nuevas medidas prolongó el interés de los lectores sobre temas da asuntos públicos, con lo que se puede explicar el achicamiento de la brecha de temas de asuntos públicos durante las últimas semanas del año. En este escenario, observamos que durante diciembre el interés del público en temas como el nuevo gabinete, la asunción del presidente Macri y el levantamiento del cepo al dólar ${ }^{15}$ hizo que el porcentaje de noticias de asuntos públicos en las noticias más cliqueadas fuera mayor que en la página de inicio en cinco de los siete sitios analizados (Clarín, La Nación, Perfil, Infobae y TN). El "pico" de interés sobre asuntos públicos por parte de los lectores se dio en noviembre y diciembre en todos los sitios excepto en Perfil (que tuvo su pico en enero) y diario Uno de Mendoza (que tuvo su pico en marzo) (cuadros 1 y 2). ${ }^{16}$

No todos los acontecimientos de asuntos públicos capturaron el interés de la audiencia. Existió una brecha significativa en la cobertura y el consumo de noticias de uno de los casos policiales más famosos de la Argentina de los últimos tiempos: la fuga de los tres condenados por el asesinato de tres empresarios en una trama de narcotráfico y política, que fue conocida en la Argentina como el "triple crimen". En una muestra de cinco días de los diecisiete que estuvieron prófugos, ${ }^{17}$ la cobertura de los medios

15 Durante los años finales de la presidencia de Cristina Fernández de Kirchner los ciudadanos podían comprar una cantidad limitada de moneda extranjera. Esta restricción fue denominada "cepo al dólar".

16 Esto se debe a que las noticias sobre una posible huelga docente en febrero-marzo y la visita del presidente de los Estados Unidos, Barack Obama, en marzo estuvieron en repetidas oportunidades entre las más leídas de ese sitio.

17 Entre el 27 de diciembre de 2015 y el 12 de enero de 2016. 


\section{Cuadro 2. Porcentaje de noticias de asuntos públicos en las listas de periodistas y consumidores entre enero y marzo de 2016}

\begin{tabular}{|c|c|c|c|c|c|c|}
\hline & \multicolumn{2}{|c|}{ Enero } & \multicolumn{2}{|c|}{ Febrero } & \multicolumn{2}{|c|}{ Marzo } \\
\hline & Periodistas & Consumidores & Periodistas & Consumidores & Periodistas & Consumidores \\
\hline \multirow[t]{2}{*}{ Clarín } & $77.94 \%$ & $55.71 \%$ & $61 \%$ & $41.43 \%$ & $87 \%$ & $63.77 \%$ \\
\hline & \multicolumn{2}{|c|}{$\begin{array}{c}\operatorname{chi} 2(1)=7.6683 \\
\operatorname{Pr}=0.006\end{array}$} & \multicolumn{2}{|c|}{$\begin{array}{c}\operatorname{chi} 2(1)=5.6046 \\
\operatorname{Pr}=0.018\end{array}$} & \multicolumn{2}{|c|}{$\begin{array}{c}\operatorname{chi} 2(1)=10.2751 \\
\operatorname{Pr}=0.001\end{array}$} \\
\hline \multirow[t]{2}{*}{ La Nación } & $87.14 \%$ & $80 \%$ & $62.86 \%$ & $71.43 \%$ & $86.96 \%$ & $68.57 \%$ \\
\hline & \multicolumn{2}{|c|}{$\begin{array}{c}\operatorname{chi} 2(1)=1.9695 \\
\operatorname{Pr}=0.160\end{array}$} & \multicolumn{2}{|c|}{$\begin{array}{c}\operatorname{chi} 2(1)=0.7609 \\
\operatorname{Pr}=0.383\end{array}$} & \multicolumn{2}{|c|}{$\begin{array}{c}\operatorname{chi} 2(1)=5.0533 \\
\operatorname{Pr}=0.025\end{array}$} \\
\hline \multirow[t]{2}{*}{ Perfil } & $80 \%$ & $96 \%$ & $80.00 \%$ & $85.71 \%$ & $92.86 \%$ & $76.67 \%$ \\
\hline & \multicolumn{2}{|c|}{$\begin{array}{c}\operatorname{chi} 2(1)=0.6026 \\
\operatorname{Pr}=0.438\end{array}$} & \multicolumn{2}{|c|}{$\begin{array}{c}\operatorname{chi} 2(1)=0.8046 \\
\operatorname{Pr}=0.370\end{array}$} & \multicolumn{2}{|c|}{$\begin{array}{c}\operatorname{chi} 2(1)=6.7863 \\
\operatorname{Pr}=0.009\end{array}$} \\
\hline \multirow[t]{2}{*}{ Infobae } & $74 \%$ & $45 \%$ & $60.00 \%$ & $45.00 \%$ & $79.03 \%$ & $66.67 \%$ \\
\hline & \multicolumn{2}{|c|}{$\begin{array}{c}\operatorname{chi} 2(1)=9.4342 \\
\operatorname{Pr}=0.002\end{array}$} & \multicolumn{2}{|c|}{$\begin{array}{c}\operatorname{chi} 2(1)=2.2451 \\
\operatorname{Pr}=0.134\end{array}$} & \multicolumn{2}{|c|}{$\begin{array}{c}\operatorname{chi} 2(1)=1.9150 \\
\operatorname{Pr}=0.166\end{array}$} \\
\hline \multirow[t]{2}{*}{$\mathrm{TN}$} & $63.77 \%$ & $12.31 \%$ & $65.71 \%$ & $17.19 \%$ & $76.67 \%$ & $17.86 \%$ \\
\hline & \multicolumn{2}{|c|}{$\begin{array}{c}\operatorname{chi} 2(1)=37.3247 \\
\operatorname{Pr}=0.000\end{array}$} & \multicolumn{2}{|c|}{$\begin{array}{c}\operatorname{chi} 2(1)=32.2092 \\
\operatorname{Pr}=0.000\end{array}$} & \multicolumn{2}{|c|}{$\begin{array}{c}\operatorname{chi} 2(1)=40.1193 \\
\operatorname{Pr}=0.000\end{array}$} \\
\hline \multirow[t]{2}{*}{$\mathrm{La} \mathrm{Voz}$} & $45.71 \%$ & $33.33 \%$ & $35.29 \%$ & $25.00 \%$ & $68.83 \%$ & $42.50 \%$ \\
\hline & \multicolumn{2}{|c|}{$\begin{array}{c}\operatorname{chi} 2(1)=1.5838 \\
\operatorname{Pr}=0.208\end{array}$} & \multicolumn{2}{|c|}{$\begin{array}{c}\operatorname{chi} 2(1)=1.2373 \\
\operatorname{Pr}=0.266\end{array}$} & \multicolumn{2}{|c|}{$\begin{array}{c}\operatorname{chi} 2(1)=7.5942 \\
\operatorname{Pr}=0.006\end{array}$} \\
\hline \multirow[t]{2}{*}{ Uno } & $46.38 \%$ & $16.67 \%$ & $42 \%$ & $27 \%$ & $47 \%$ & $37 \%$ \\
\hline & \multicolumn{2}{|c|}{$\begin{array}{c}\operatorname{chi} 2(1)=9.0426 \\
\operatorname{Pr}=0.003\end{array}$} & \multicolumn{2}{|c|}{$\begin{array}{c}\operatorname{chi} 2(1)=2.3308 \\
\operatorname{Pr}=0.127\end{array}$} & \multicolumn{2}{|c|}{$\begin{array}{c}\operatorname{chi} 2(1)=0.9479 \\
\operatorname{Pr}=0.330\end{array}$} \\
\hline
\end{tabular}

sobre el tema fue sensiblemente mayor que el interés de sus audiencias sobre él. En Clarín, La Nación y Perfil, más de una de cada tres de las noticias de sus respectivas páginas de inicio fue sobre los prófugos. Entre las noticias más leídas en esos sitios, apenas una de cada ocho en Clarín y La Nación, y una de cada cinco en el caso de Perfil fueron sobre ese tema. En el resto de los sitios, tanto la cobertura como el consumo fueron menores. El interés relativamente bajo del público por el tema de los prófugos del triple crimen ocurre durante un mes signado por el fin de una larga campaña, la asunción de un nuevo Gobierno y un cambio fuerte en el interés del público al inicio de la temporada de vacaciones de verano (enero).

En general, los dos meses subsiguientes a la asunción del nuevo Gobierno, es decir, enero y febrero, volvieron a mostrar una brecha noticiosa 
más amplia; este hallazgo tiene sentido en la medida en que no solo fueron meses de baja actividad debido a las vacaciones, sino que, además, produjeron una situación de "descanso" luego de intensos meses de actividad política y elecciones. Esto puede verse claramente no solo con el bajo interés de los lectores en el caso de la "triple fuga", sino también al observar los datos en marzo: durante este mes vemos que el interés de la audiencia en temas de asuntos públicos vuelve a aumentar. Tal hecho puede explicarse, en cierta medida, por la vuelta a la actividad política e institucional luego de los meses de verano (apertura de sesiones en el poder legislativo, comienzo de clases y negociaciones salariales, etc.). Otro hecho que ayuda a explicar el aumento de atención en temas de asuntos públicos tiene que ver con la visita del presidente Barack Obama a fines de marzo, durante la cual, aproximadamente, casi tres de cada diez de las primeras noticias de la página de inicio del sitio cubrieron su gira y alrededor de un cuarto de las más cliqueadas fueron sobre el tema.

Si bien hay variaciones por sitio (cuadros 1 y 2 ) en la mayoría es evidente una brecha entre las preferencias de periodistas y consumidores que se achicó o desapareció en noviembre y diciembre de 2015 (en La Voz fue a partir de octubre, en Perfil no hubo brecha hasta marzo de 2016). En La Nación, la desaparición de la brecha se extendió hasta febrero de 2016.

Este análisis indica que el interés por temas de asuntos públicos aumenta durante los periodos de mucha actividad política, como hemos visto en el caso de la campaña electoral de 2015, sumada a las atracciones de una segunda vuelta sin ganador definido y la expectativa generada ante la asunción de un nuevo Gobierno.

\section{Discusión}

Los hallazgos presentados en este trabajo indican que, excepto en diciembre de 2015, entre septiembre de 2015 y marzo de 2016, hubo una brecha entre las noticias que los periodistas eligieron publicar de manera más prominente en la homepage y los artículos que los consumidores seleccionaron: mientras los productores de noticias privilegiaron los temas de política nacional e internacional y economía y negocios, el público tendió 
a preferir artículos sobre temas no públicos, como deportes, espectáculos y policiales. Por otro lado, la selección de temas de los periodistas fue más estable que la de los integrantes del público: el porcentaje más bajo de noticias sobre asuntos públicos fue $54 \%$, en septiembre de 2015 , y el más alto, $77 \%$, dos meses después. En cambio, el porcentaje más bajo de este tipo de noticias en la lista de las más leídas fue $32 \%$ en septiembre y $68 \%$ en noviembre, mes de la segunda vuelta presidencial. Esto llevó a una oscilación de 23 puntos porcentuales en el caso de los medios y de 36 puntos porcentuales en el caso de los periodistas.

El análisis empírico ofrece tres hallazgos principales. Primero, existe una brecha consistente entre las preferencias de los periodistas y los consumidores de siete sitios en línea en la Argentina durante un periodo que incluye una campaña presidencial y la fuga de la cárcel y posterior detención de tres condenados por tres homicidios con fuertes conexiones con el poder político. Segundo, hay una mayor variabilidad en las preferencias de los integrantes del público que en las elecciones de los productores profesionales de noticias. Tercero, se registra una notable excepción, diciembre de 2015, en el que la brecha entre periodistas y consumidores desaparece.

Estos tres hallazgos nos permiten reflexionar sobre el rol de la oferta y la demanda de noticias en la matriz de política y comunicación de la segunda década del siglo XXI. La brecha confirma que periodistas y editores, tanto por formación profesional (Cook, 1998; Davis 1995; Williams y Delli Carpini, 2012) como por organización del trabajo (Bennett, Lawrence y Livingston 2007; Gitlin, 1980), tienden a privilegiar noticias sobre asuntos públicos en todo momento. En cambio, las audiencias de sitios de noticias en línea tienden a seleccionar menos noticias sobre asuntos públicos y a cambiar sus elecciones informativas de manera más variable que la oferta de los medios (Bird, 2003; Hamilton, 2004; Prior, 2007; Tewksbury, 2003). Esta preferencia se debe a que las noticias sobre temas "livianos", como espectáculos o deportes, demandan menos esfuerzos cognitivos — es decir, son más fáciles de comprender por la mayoría de los integrantes del públicoy generan estados afectivos más positivos a los consumidores de noticias (Boczkowski y Mitchelstein, 2013). 
La mayor variabilidad en las preferencias de los integrantes del público que en la de los periodistas indica que los consumidores de noticias se comportan, en alguna medida, como ciudadanos monitoriales (Schudson, 1998; Zaller, 2003), y aumenta su atención a temas de asuntos públicos en momentos de mayor actividad política, como una elección presidencial en la que los dos candidatos principales se encuentran muy parejos o la asunción de un nuevo Gobierno. Aunque el esfuerzo cognitivo que demanda esta información puede ser alto, esto es contrarrestado por dos factores que impulsan la atención a noticias de asuntos públicos. En primer lugar, saber quién es el ganador de una elección presidencial, cómo conforma su gabinete de ministros y cuáles son sus primeras medidas puede afectar directamente la vida de los ciudadanos, que consumen esta información por un sentimiento de autopreservación. Según Shoemaker y Cohen (2006), prestamos atención a las noticias que podrían afectar directamente nuestras vidas, como la asunción de un nuevo Gobierno, debido al impulso evolutivo de preservarnos y adaptarnos a nuestro entorno para sobrevivir. En segundo lugar, un nuevo Gobierno puede generar sensaciones positivas en las audiencias, lo cual las impulsaría a consumir las noticias sobre la asunción del presidente y sus primeras medidas. Como contraejemplo, la noticia sobre la triple fuga no solo era un tema complejo, sino que además no era importante para la mayoría de los consumidores, y tendía a despertar estados afectivos negativos.

La excepción de diciembre, mes en el que no hubo brecha entre editores y consumidores, paradójicamente, marca los límites a la fijación de agenda por parte de los medios periodísticos. La teoría de fijación de agenda (McCombs y Shaw, 1972) indica que los medios no le dicen a la ciudadanía qué pensar, pero sí sobre qué temas deben pensar (Cohen, 1963). Si bien eso se puede haber cumplido cuando asumió un presidente de signo político distinto del anterior por primera vez en catorce años, no sucedió lo mismo durante el resto del periodo de siete meses estudiado. En el nuevo entorno de comunicación, en el que los consumidores pueden seguir con mayor libertad sus preferencias (tanto por la mayor variedad de medios entre los cuales elegir como por las mayores posibilidades de seleccionar contenido dentro de esos medios), aunque los productores profesionales de 
noticias puedan sugerir una agenda, esa agenda no necesariamente será tomada por el público como la agenda de temas que están obligados a seguir.

La existencia de una brecha, sumada a las mayores facilidades para que el público pueda ejercer la ciudadanía monitorial y a la pérdida en el poder de fijación de agenda de los medios, pone en cuestión las estructuras de información y comunicación que dominaron la producción de noticias y la política durante gran parte del siglo XX. El surgimiento de nuevos competidores de los medios periodísticos tradicionales y la mayor libertad para las audiencias sugieren que el modelo de comunicación en el que actores poderosos de la política, las empresas y la sociedad civil le hablaban al público - y lograban captar su atención — a través de un número limitado de fuentes de información ya no es tan efectivo. Esperamos que esta investigación sea un primer paso en la dirección hacia encontrar nuevas maneras de comunicarnos entre ciudadanos.

\section{Referencias}

Bennett, W. L. y Entman, R. M. (2001). Mediated politics: An introduction. En L. Bennett y R. Entman (Ed.), Mediated politics: Communication in the future of democracy (pp. 1-20). Nueva York: Cambridge University Press.

Bennett, W. L., Lawrence, R. G. y Livingston, S. (2008). When the press fails: Political power and the news media from Iraq to Katrina. Chicago: University of Chicago Press.

Bird, S. E. (2003). The audience in everyday life: Living in a media world. Nueva York: Routledge.

Boczkowski, P. J., Mitchelstein, E. y Walter, M. (2012). When burglar alarms sound, do monitorial citizens pay attention to them? The online news choices of journalists and consumers during and after the 2008 US election cycle. Political Communication, 29(4), 347-366. 
Boczkowski, P. J. y Mitchelstein, E. (2013). The news gap: When the information preferences of the media and the public diverge. Cambridge, Londres: The MIT Press.

Cho, J. (2008). Political ads and citizen communication. Communication Research, 35(4), 423-451.

Cohen, B (1963). The press and foreign policy. Nueva York: Harcourt.

Cook, T. E. (1998). Governing with the news: The news media as a political institution. Chicago, Londres: University of Chicago Press.

Davis, R. (1995). The press and american politics: The new mediator. Londres: Longman.

Davis, A. (2000). Public relations, news production and changing patterns of source access in the British national media. Media, Culture do Society, 22(1), 39-59.

Dean, W., Pertilla, A. y Belt, T. (2007). The myths that dominate local TV news: The X-structure, and the fallacy of the hook-and-hold method of TV news. En T. Rosenstiel, M. Just, T. Belt, A. Pertilla, W. Dean y D. Chinni (Eds.), We interrupt this newscast: How to improve local news and win ratings, too. Nueva York: Cambridge University Press.

Downs, A. (1957). An economic theory of Democracy. Nueva York: Harper \& Row.

Druckman, J. N. (2005). Media matter: How newspapers and television news cover campaigns and influence voters. Political Communication, 22(4), 463-481.

Gans, H. J. (2004). Deciding what's news: A study of CBS evening news, NBC nightly news, Newsweek, and Time. Chicago: Northwestern University Press. 
Gitlin, T. (1980). The whole world is watching: Mass media in the making and unmaking of the new left. Berkeley: University of California Press.

Graber, D. A. (2006). Government by the people, for the people-twentyfirst century style. Critical Review, 18(1-3), 167-178.

Hamilton, J. (2004). All the news that's fit to sell: How the market transforms information into news. Princeton: Princeton University Press.

Huang, E. (2009). The causes of youths' low news consumption and strategies for making youths happy news consumers. Convergence, 15(1), 105-122.

Instituto Verificador de Diarios y Revistas (IVC) (2016). El Boletín.

Iyengar, S. y Kinder, D. R. (1987). News that matters: Agenda setting and priming in a television age. Chicago, Londres: University of Chicago Press.

Jones, A. (2009). Losing the news: The future of the news that feeds democracy. Oxford, Nueva York: Oxford University Press.

McCombs, M. E. y Shaw, D. L. (1972). The agenda-setting function of mass media. Public Opinion Quarterly, 36(2), 176-187.

McCombs, M. y Stroud, N. J. (2014). Psychology of agenda-setting effects: Mapping the paths of information processing. Review of Communication Research, 2(1), 68-93.

Patterson, T. E. (1994). Out of order: An incisive and boldly original critique of the news media's domination of America's political process. Nueva York: Vintage.

Prior, M. (2007). Post-broadcast democracy: How media choice increases inequality in political involvement and polarizes elections. Nueva York: Cambridge University Press. 
Scheufele, D. A. y Tewksbury, D. (2007). Framing, agenda setting, and priming: The evolution of three media effects models. Journal of Communication, 57(1), 9-20.

Schudson, M. (1978). Discovering the news: A social history of American newspapers. Nueva York: Basic Books.

Schudson, M. (1998). The good citizen: A history of American civic life. Nueva York: Martin Kessler Books.

Shoemaker, P. J. y Cohen, A. A. (2006). News around the world: Content, practitioners, and the public. Nueva York, Londres: Routledge.

Tewksbury, D. (2003). What do americans really want to know? Tracking the behavior of news readers on the Internet. Journal of Communication, 53(4), 694-710.

Tewksbury, D. (2006). Exposure to the newer media in a presidential primary campaign. Political Communication, 23(3), 313-332.

Tuchman, G. (1978). Making news: A study in the construction of reality. London, Nueva York: Free Press.

Williams, B. A. y Carpini, M.X. D. (2011). After broadcast news: Media regimes, democracy, and the new information environment. Nueva York: Cambridge University Press.

Zaller, J. (2003). A new standard of news quality: Burglar alarms for the monitorial citizen. Political Communication, 20(2), 109-130.

Zelizer, B. (2004). Taking journalism seriously: News and the academy. Thousand Oaks: Sage Publications. 